\title{
Morphological and mechanical investigation of interconnected gridshells
}

\author{
Tim-Joachim Jensen ${ }^{12}$, Olivier Baverel ${ }^{13}$, Cyril Douthe $^{4}$ \\ ${ }^{1}$ Université Paris-Est, Laboratoire Navier, ENPC-IFSTTAR-CNRS, UMR 8205, France \\ ${ }^{2}$ ENSAV, France \\ ${ }^{3}$ ENSAG, France \\ ${ }^{4}$ Université Paris-Est, IFSTTAR.
}

\begin{abstract}
This paper describes the structural reinforcement of grid shells by interconnecting several gridshells together. Reference for the present investigation is taken from the composite (GFRP) gridshell structure built for the Forum of the Solidays music festival in Paris in 2011. The principle of interconnection is illustrated with various structures which are generated using a grasshoppper components developed by the Navier laboratory for the automatic meshing of free-forms structures on Rhino. Then the effect of the interconnection on the structural behaviour is briefly discussed based on elementary loading cases. Finally, the feasibility of the concept as well as its architectural and morphological potential is show by the construction of a double gridshell prototype. The authors conclude on the possible future developments of such morphologies which could give interesting and unexpected architectures.
\end{abstract}

\section{Introduction}

The Navier laboratory has participated to the design and construction of the first composite (GFRP) gridshell building for a public event, the Solidays music Festival in june 2011 (see figure 1), sponsored by academic (ENPC), caritative (Solidarité Sida) and industrial partners (T/E/S/S, Viry) [1]. It was made possible with a team that has worked on (GFRP) gridshell knowledge for about ten years [2-5]. Speaking of structural types, the word gridshell commonly describes a grid structure having the shape and the rigidity of a double curvature shell. In the present case, the word gridshell will be used for elastic gridshell meaning there that the form is obtained by elastic deformation of a two-way grid initially flat. At the end of the forming process, the deformed grid is rigidified, using presently a third direction of beams.

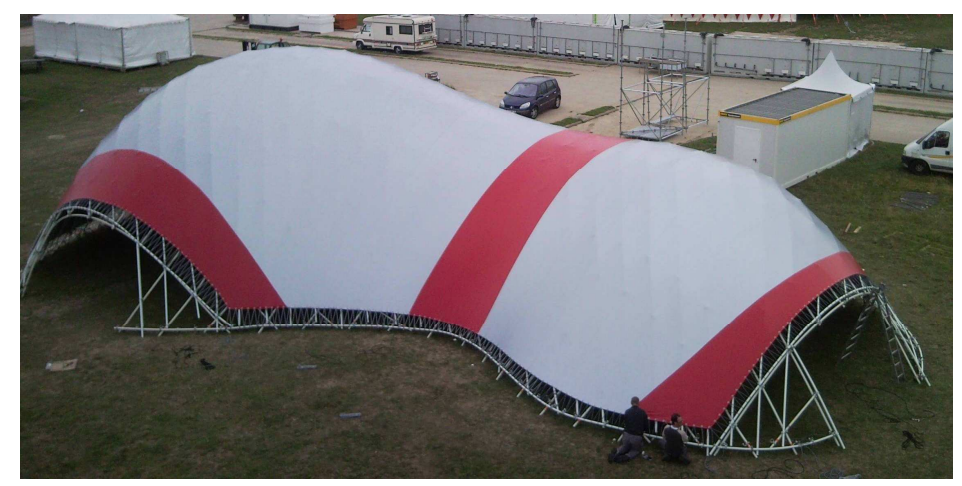

Figure 1: Aerial view of the gridshell for the Solidays music festival (2011) 
In this paper, a morphological investigation of several gridshells that intersect each other is presented, originally aiming at improving the structural behaviour of such structures. The gridshell built for the Solidays festival is taken as a reference for the various interconnected gridshells with similar spans which will be proposed. In the following section, the numerical tools used to adjust the form, to take into account the mechanical constrains and the method to mesh the form are presented. In the third section then, different cases of interconnected gridshells are shown and key issues of their mechanical behaviour are illustrated by comparison with the configuration of the Solidays structure. In the fourth section, the feasibility of the concept of two interconnected gridshells is practically studied by constructing a prototype during a workshop organized with the master students.. Finally, the morphological potential is illustrated before concluding on the general advantages of such configurations.

\section{Experimental Feedback on the Solidays Building}

As a guideline, the designer has to concentrate his efforts on three important points:

- That the curvature in each bar is not too high;

- That the entire surface is meshed;

- That the mesh does not get too concentrated locally.

\subsection{Global and local transformation of the surface}

Figure 2 shows the first drawing steps with the possible transformation of the freeform surface which must be meshed on Rhino and which actually ressemble a half-peanut with two domes and a curvature inversion between them. This surface is defined as a patch on the contour polylines (visible on figure 2 (A) which defines the perimeter in plan and on some contour polylines in elevation which defines the height of the building [6]. The patching command depends on a stiffness parameter which govern the smoothing of the surface. For the surface in figure 2, the parameters are the following: Stiffness, k=5, Surface U Spans=10, Surface V Spans=10; (the red line are the support curves for the patch). If necessary, this surface can be modified locally by displacing the surface control points according to the designer wishes (see figure 2, B). It must be noted here that if the sample point spacing of the patch surface is too high, the manipulation of the control points gets too precise and inappropriate for the definition of a smooth surface.

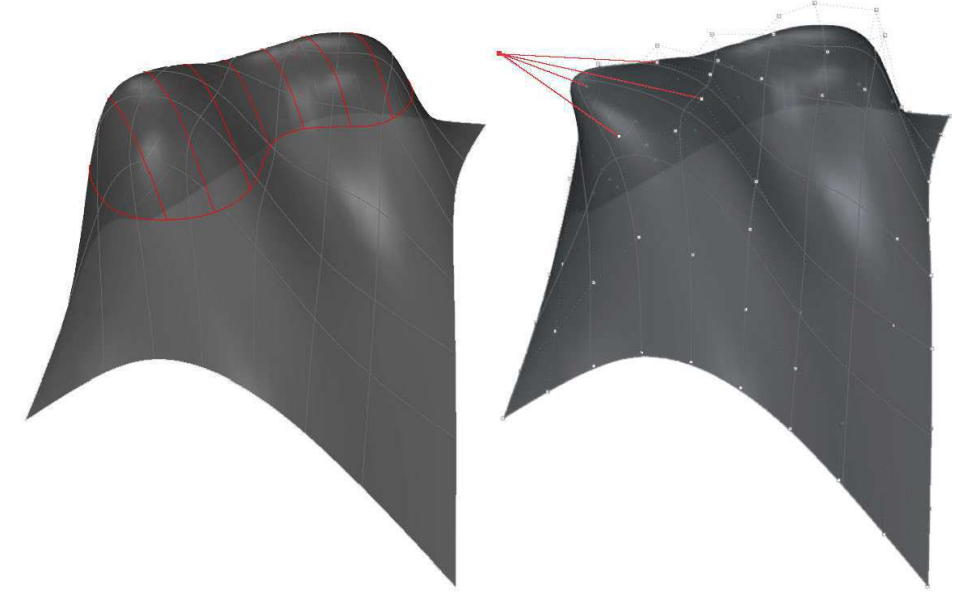

Figure 2: Aerial view of the patch stiffness $(A)$ and the control point transformation $(B)$ 


\subsection{Geometrical constraints due to the structural behaviour}

The main stresses in the gridshell are due to the bending in the beams due to the forming process (not to variable external loads) [3-4]. The geometry of the surface to be meshed imposes thus the stress distribution in the gridshell members. These stresses are inversely proportional to the curvature and are given by the expression:

$$
\sigma=\sigma_{\text {comp }}+\sigma_{\text {flex }} \approx \sigma_{\text {flex }}=\frac{E \cdot v}{R}
$$

where $\sigma$ denotes the stress in $\mathrm{MPa}, \mathrm{E}$ the Young modulus in $\mathrm{MPa}, \mathrm{v}$ the distance to the neutral axis of the member in $\mathrm{m}$ and $\mathrm{R}$ its radius of curvature in $\mathrm{m}$. For a given material (here the GFRP tubes used for the Solidays structure) this stress must remain below a certain limit $\left(\sigma_{\max }=200 \mathrm{MPa}\right)$. Thus for a given section (here a $48 \mathrm{~mm}$ diameter tube), the above equation can be transformed into a geometrical constrain:

$$
E \cdot \frac{V}{R_{\min }} \leq \sigma_{\max } \Rightarrow R_{\min } \geq \frac{E \cdot v}{\sigma_{\max }}(=3.28 m)
$$

As the extreme radii of curvature $R_{\min }$ and $R_{\max }$ can be estimated easily by Rhino, it is possible to handle the surface in order to keep its curvature below a certain level insuring hence that a mesh will be feasible. Indeed figure 3 shows a comparison between stresses in the grid and curving radii of the surface with $R_{\min }=3 \mathrm{~m}$ and $\left.R_{\max }=8.7 \mathrm{~m}\right)$... In figure $3(\mathrm{~A})$, the local stress (in red) located in the neighboring of the doors must be disregarded as local strengthening have been added in those parts. This being said, it can be remarked that the most loaded member are submitted to stresses between 100 and 140MPa. These members are located on the blue regions of the curvature diagram (figure 3(B)) which corresponds to where the lowest radii. This observation is a good starting point for the design of the surface but does not presume of the mechanical behavior of the structure under self-weight or external forces which must still be investigated. It just can be guessed that the most flat areas (in red on figure 3(B)) will form the weakest zones of the structure when loaded.
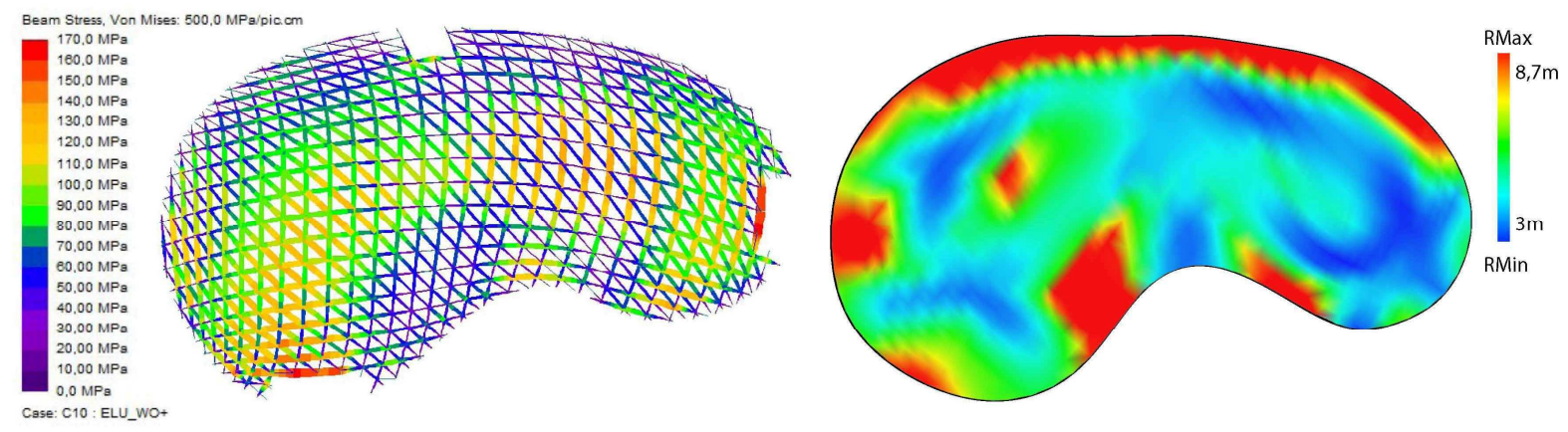

Figure 3: Comparison between the prestresses in the grid $(A)$ and the minimum radii of the surface $(B)$

\subsection{Meshing of the Surface}

The "compass method" used to generate the grid consists in constructing a network of parallelograms on a given surface. This method was described in IL10 Gitterschalen of Frei Otto et al [7]. Figure 4 shows the different steps of the method on a plane surface. The task is to construct a grid of parallelograms on a surface with a compass. Two arbitrary intersecting curves or generators are first laid on the surface. Then, a mesh width is selected and serves as compass radius. The spacing of the grid is marked along each axis, starting from the point of intersection of the axes. Afterwards, the nodes are determined by the intersection of two 
circles drawn around each of the neighboring points. Then, gradually, new points are determined in the same way. Finally the nodes are connected rectilinearly.

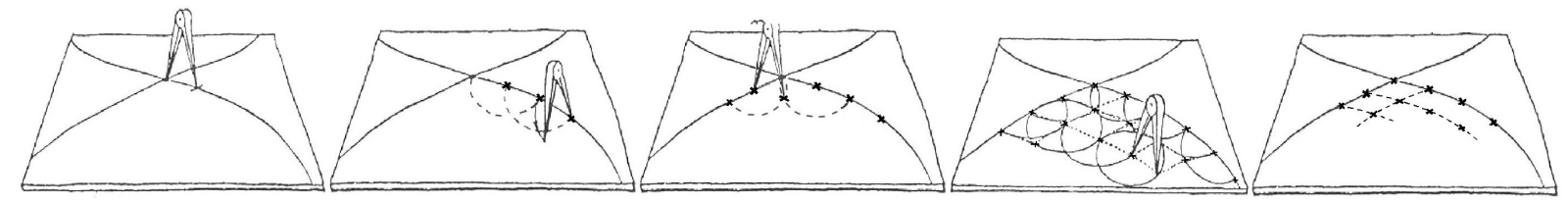

Figure 4: Principle of “compass method” (after IL10 [7])

For non plane surfaces, the principle of the construction of the grid is exactly the same than previously. This method leads to a grid that fits the 3D surface and allows hence in a certain way for the "development" of non-developable surfaces. It has been implemented numerically in grasshopper for Rhino to mesh free forms and an illustration of it is shown in figure 5 (with in dark grey the patch surface, in light grey the cutting surface for the meshing, in red the director polylines for the "compass method" meshing, in black the main lines of the meshing and in light grey the lines for the third/fourth direction of the meshing). It can be seeen that the position and the geometry of the director lines on the patch surface as well as the chosen step define a specific grid. The direction of these polylines should as possible follow the geometry of the form: a bad choice of this director polylines creates a nonharmonious grid or an incomplete grid (see figure 5, B) with local concentration of the mesh. Although one could think of possible automation of the generation of optimal meshes, the search for a suitable mesh is here done manually.
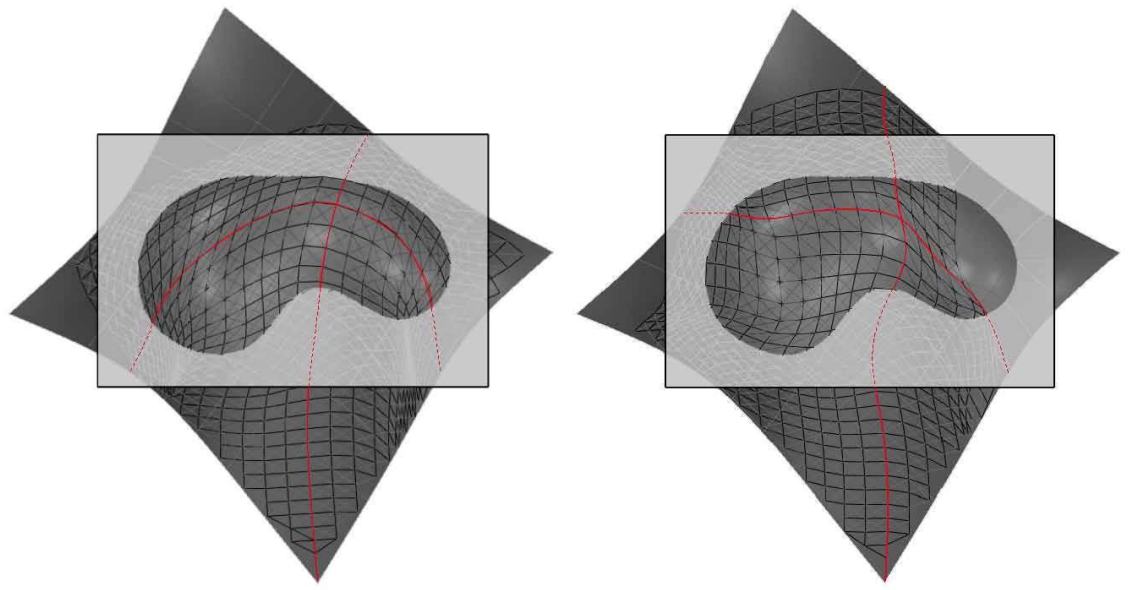

Figure 5: Complete (A) and incomplete (B) meshing of the patch surface

\subsection{Mechanical behaviour}

The mechanical behaviour of the structure has then been studied. The reference grid was generated following the compass method based on the two generators shown on figure 5A and a mesh width of $1 \mathrm{~m}$. This led to a total length of beams of approximately $1200 \mathrm{~m}$ for the main grid and $1600 \mathrm{~m}$ for the triangulation (here in both directions contrary to the real Solidays building). For the present study, the actual entrances of the structure are not considered and boundary conditions are thus simple hinged supports on the reference plane for all members extremities. Concerning loading conditions, a single representative case has been investigated: it is composed of the self weight of the structure and an equivalent snow load of approximately $40 \mathrm{daN} / \mathrm{m}^{2}$ transformed into uniform nodal forces of $25 \mathrm{daN}$ at every nodes. Two analyses are conducted (with the software Autodesk Robot Structural Analysis): 
an elastic linear analysis and a linear buckling analysis. For these two calculus, the initial bending prestress has been neglected (for simplicity reasons and also because current research of the authors tends to show that the prestress plays a secondary role in whole stability of the grid).

The results of the linear analysis are shown in figure 6 . It appears that the deformations concentrate mainly in two areas: the first one in the inner central part of the peanut, the other in the outer central part. The comparison of this figure with that of figure 3B shows that these area correspond to the two zones where the curvature is the smallest. Structural analysis hence confirms the initial intuition that flat areas constitute the weakest points of the structure.
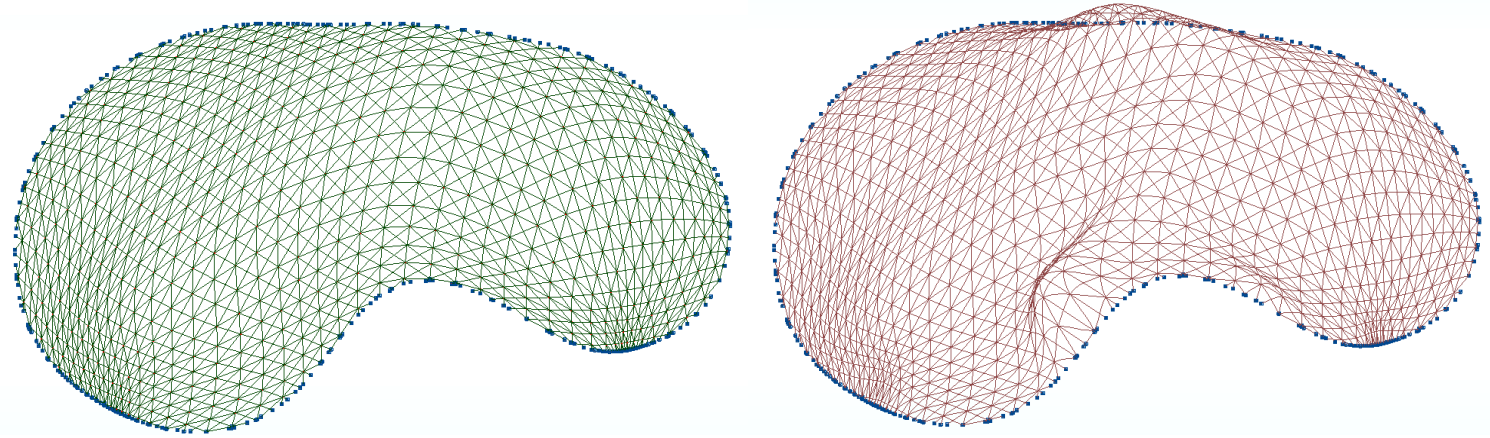

Figure 6: Initial and deformed shape of the gridshell under uniform snow load.

This intuition is also verified by the linear buckling analysis which shows that the first ten buckling modes are all located in those areas (figure 7). Modes 1 to 4 concerns the inner area $\mathrm{A}$, mode 5 zone $\mathrm{B}$, mode 6 zones $\mathrm{A}$ and $\mathrm{B}$, modes 7 and 8 zone $\mathrm{C}$ and modes 9 and 10 the three zones. Typical load factors are 5 for mode 1,10 for mode 5 and 11 for mode 7 , so that in its actual form, the structure is safe.

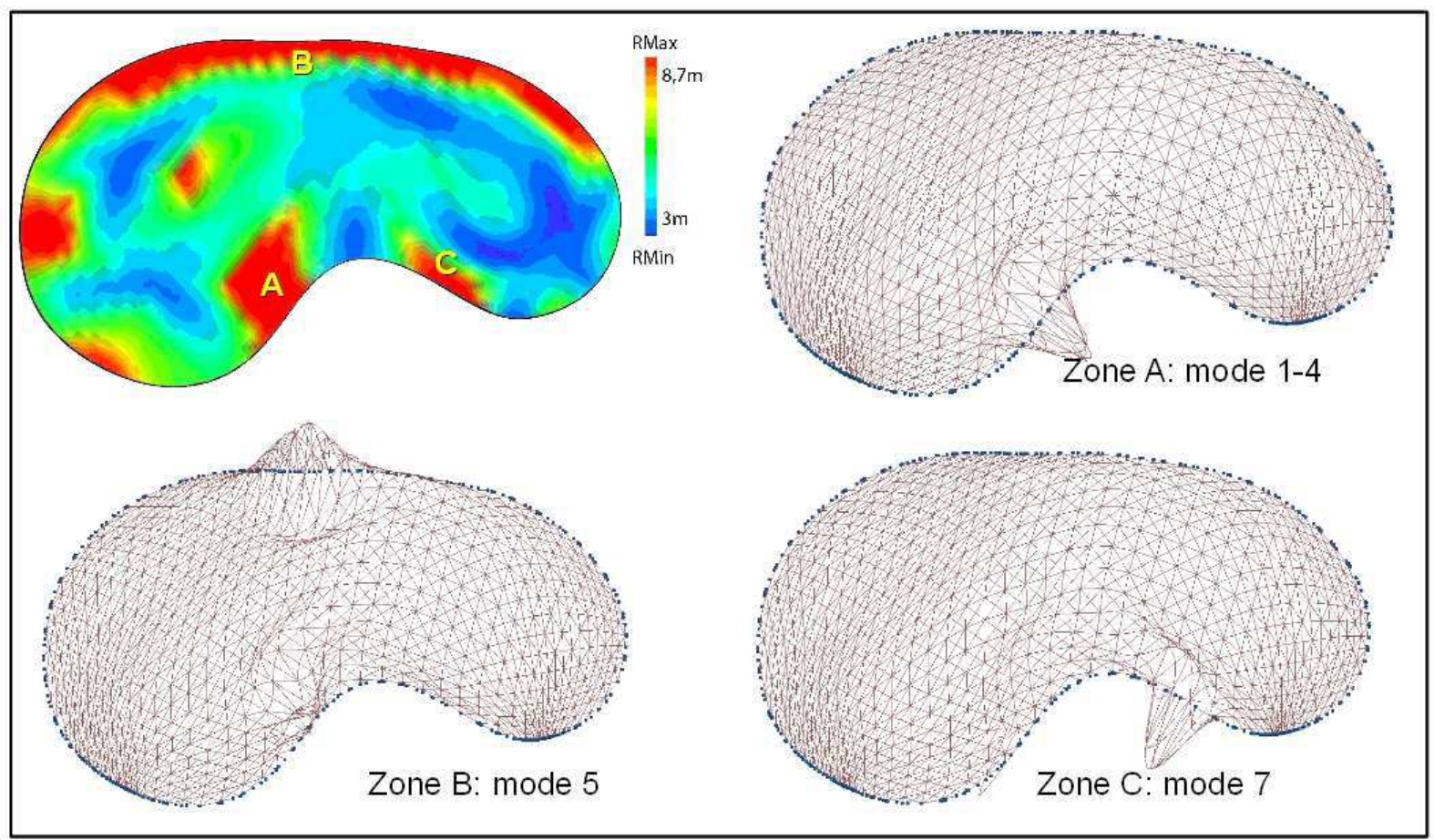

Figure 7: Buckling modes

However, one could think of strategies to improve the structural performances of this building, among which: 
- increasing the grid density (which increases self weight and the work required for the construction)

- increasing the section of the members (which is actually not a good strategy as it increases also bending prestress and may not be possible for the investigated surface without exceeding the stress limit);

- introducing local reinforcements (with for example some additional stiffer members or as will be investigated in next section by intersecting in those areas the gridshell with another gridshell...).

\section{Morphological and mechanical investigation of interconnected gridshells}

In this section, the conception of gridshells based on the addition and intersection of surfaces is presented. These intersections and additions are made using various operations presented in figure 8,11 and 14. The two meshes of these intersecting surfaces are connected along the intersecting lines with hinges similar to those used for the two main grids.

\subsection{Interconnection of two identical gridshells}

The reference patch surface and the director lines for the meshing presented in figure $5 \mathrm{~A}$ are here duplicated and translated horizontally in one direction (see figure 8B). Then the two patch surfaces and their director lines are scaled in one direction to enlarge the inner space in plan to cover approximately the same area as the reference structure. The distance between the surfaces is limited to two meters and the region of crossing is located on the highest part of each surface where the curvature is the most important. The principle of the duplication is first illustrated on a 2D cut where the black dashed line stands for the reference patch surface, the grey dashed line for the reference clone surface (identical), the black continuous line for the 1D scale of the reference patch surfaces and the black continuous line for the 1D scale of the reference clone patch surface.

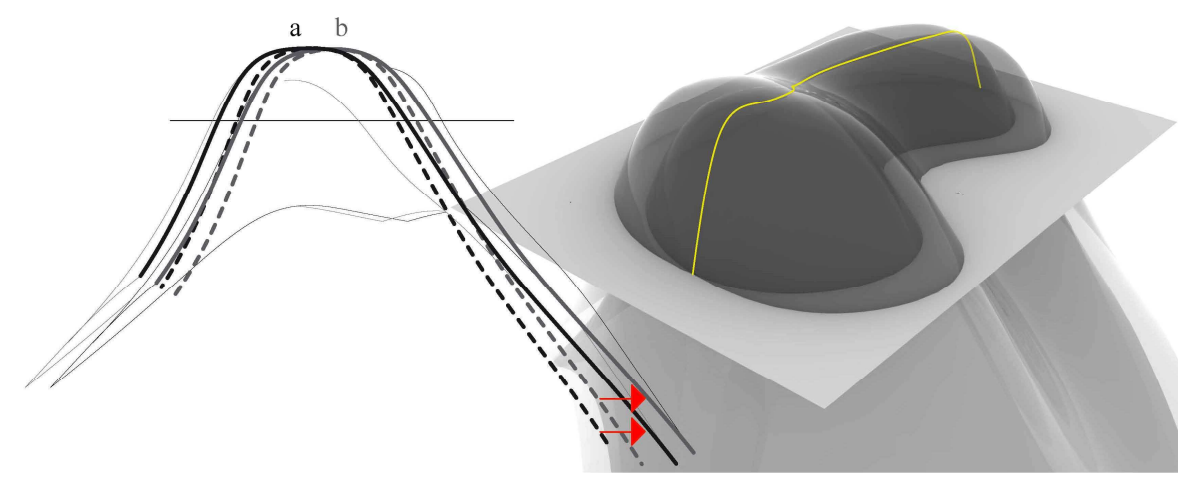

Figure 8: Cut front view (A) and aerial view (B) of the intersection of two identical surfaces

The radii of curvature of the both surfaces are then shown on figure 9. It is noted that the scaling of interconnected grids reduces the low curvature regions located on the border areas of the surface as well as the global minimum curvature of the surface. The maximum curvature radius increases at the same time. However the minimum radius value did not change because the surface is modified in only one direction. 

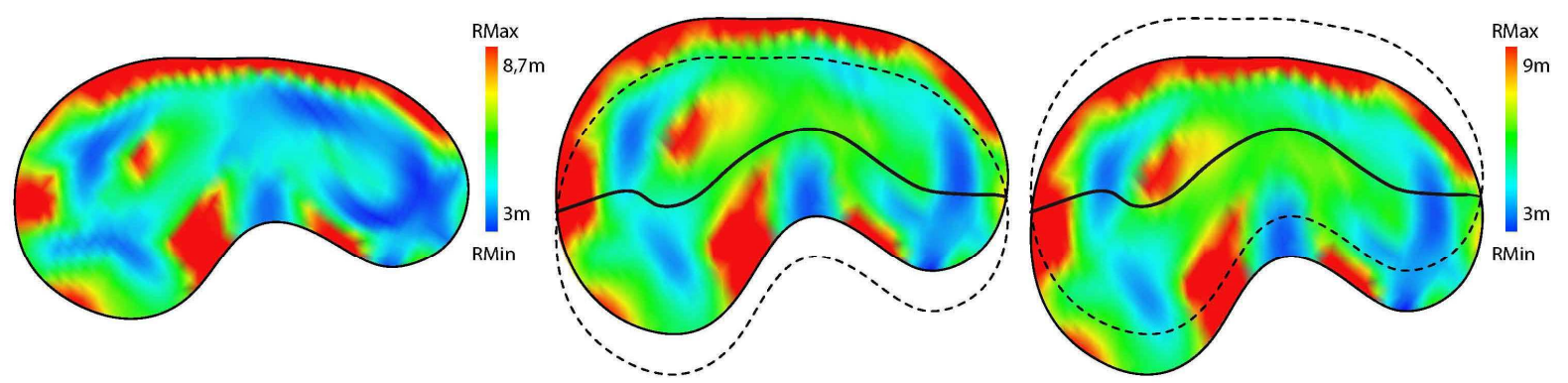

Figure 9: Comparison between the minimum radius of the reference surface and the two intersecting surfaces

Grids are then generated on the two intersecting surfaces to form the two interconnected gridshells. Figure 10 shows different views of them: (A) inside meshing plan, (B) outside meshing plan, (C) inside meshing parallel perspective, (D) outside meshing parallel perspective/(a) with in black the initial gridshell and in grey the clone gridshell.
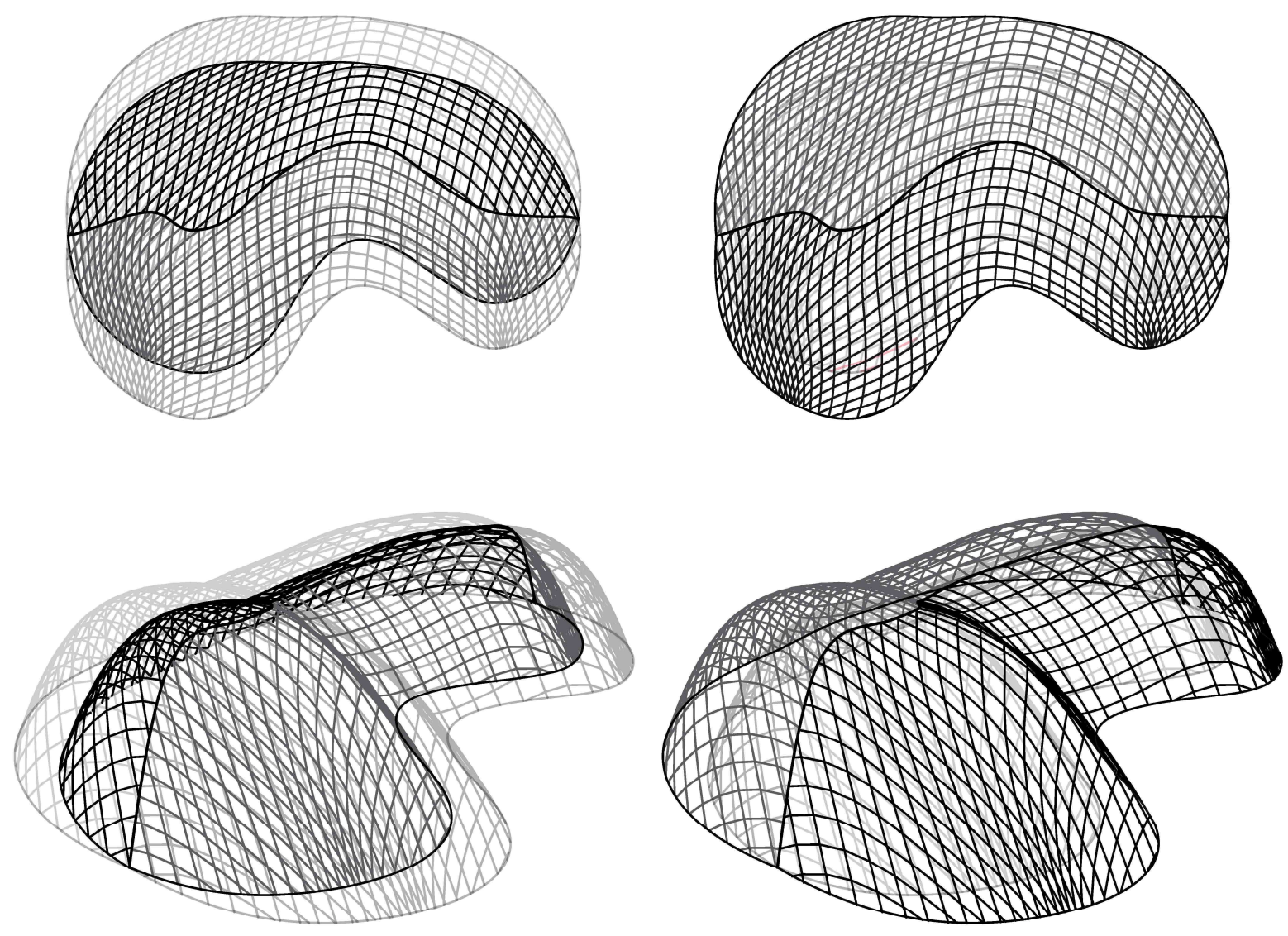

Figure 10: Meshing of the intersecting surfaces

\subsection{Interconnection of two gridshells on the same perimeter}

In this case, the patch surfaces are resulting from the transformation of the reference support curves for the patch (see figure 2A). The perimeter of the two surfaces is the reference surface perimeter. The support curves for each patch surfaces (see figure 11A) are drawn between the reference curves supporting the patch (c) and the reference offset curves (d). The distance between the two curves is limited to two meters. 


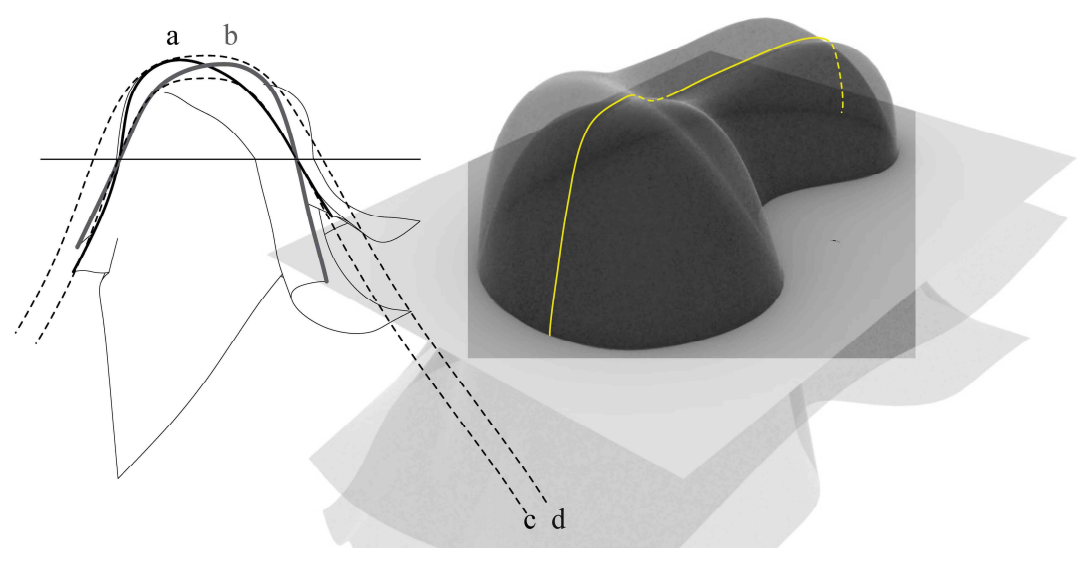

Figure 11: Cut front view (A) and Aerial view (B) of the intersection of two surfaces on the same perimeter

The high curvature on the support curves leads to a reduced minimum radius as can be seen in figure 12: initially $R_{\min }=3 \mathrm{~m}$ and $\mathrm{R}_{\max }=8.7 \mathrm{~m}$ after transformation for the first surface $R_{\min }=0.9 \mathrm{~m}$ and $R_{\max }=11.2 \mathrm{~m}$ and for the second surface $R_{\min }=1,2 \mathrm{~m} ; R_{\max }=9.7 \mathrm{~m}$.

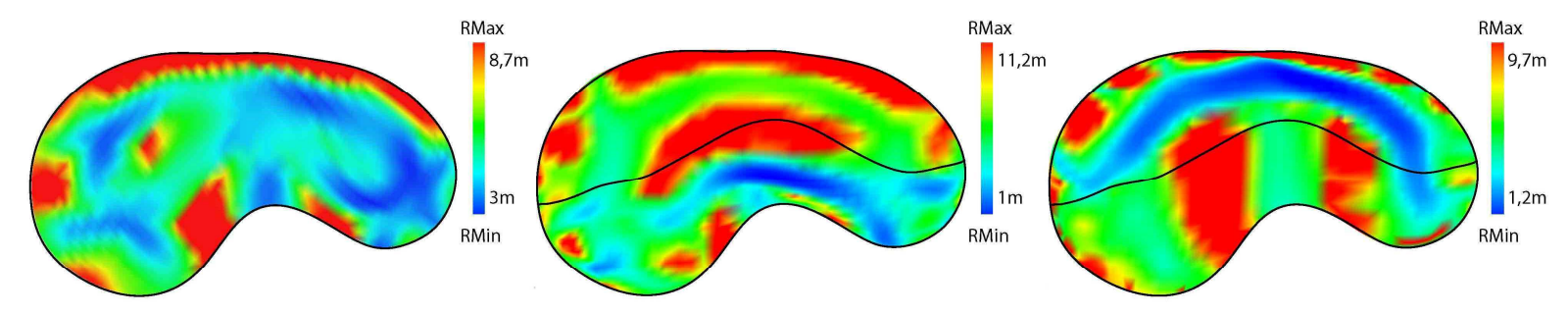

Figure 12: Comparison between the minimum radius of the reference surface and the two intersecting surfaces.

Figure 13 shows then different views of the interconnected gridshell with like previously: (A) inside meshing, (B) outside meshing, (C) inside meshing parallel perspective, (D) outside meshing parallel perspective.
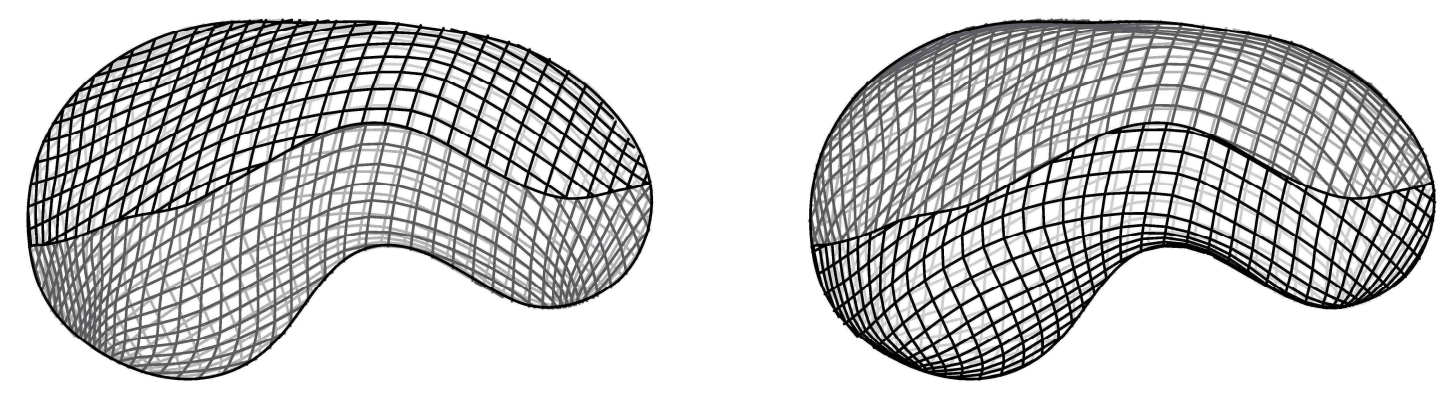

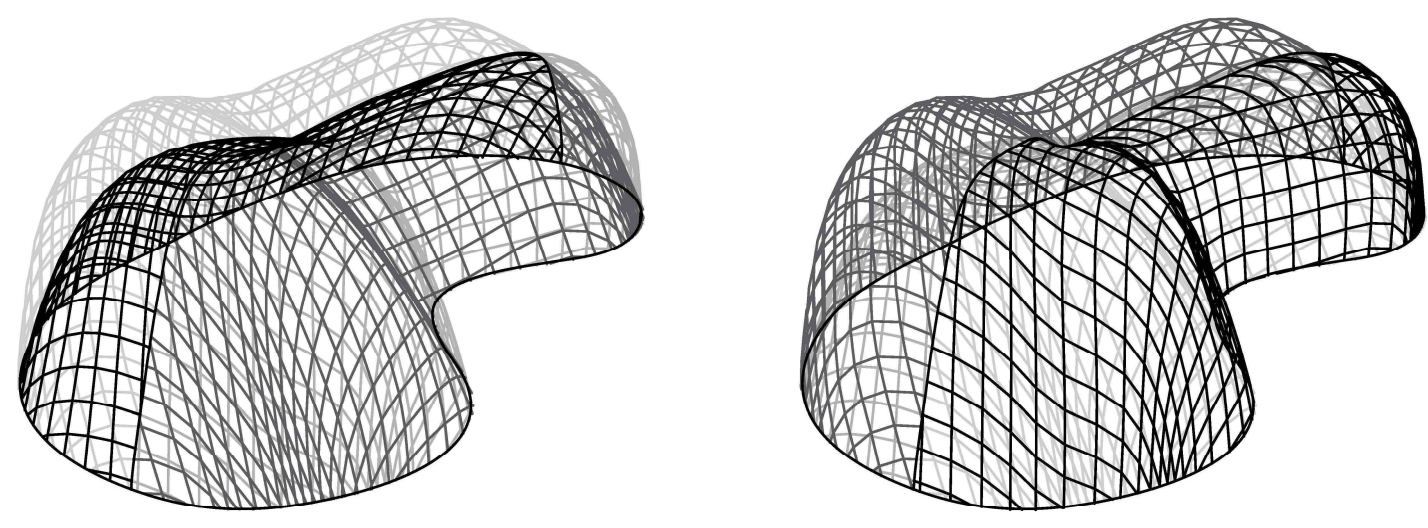

Figure 13: Meshing of the intersecting surfaces

\subsection{Interconnection of two homothetic gridshells}

For this third illustration, the reference surface is intersected with a larger surface with a lower altimetry (see figure 14). The reference patch surface (a) and the director lines for the meshing presented in figure $2 \mathrm{~A}$ are offset so that the distance between the surfaces is limited to two meters. Then the offset patch surface with the director lines is scaled in one direction vertically to intersect the reference surface. The region of crossing is located on the highest part (see figure 14) of each surface where the curvature is the most important (see figure 15).

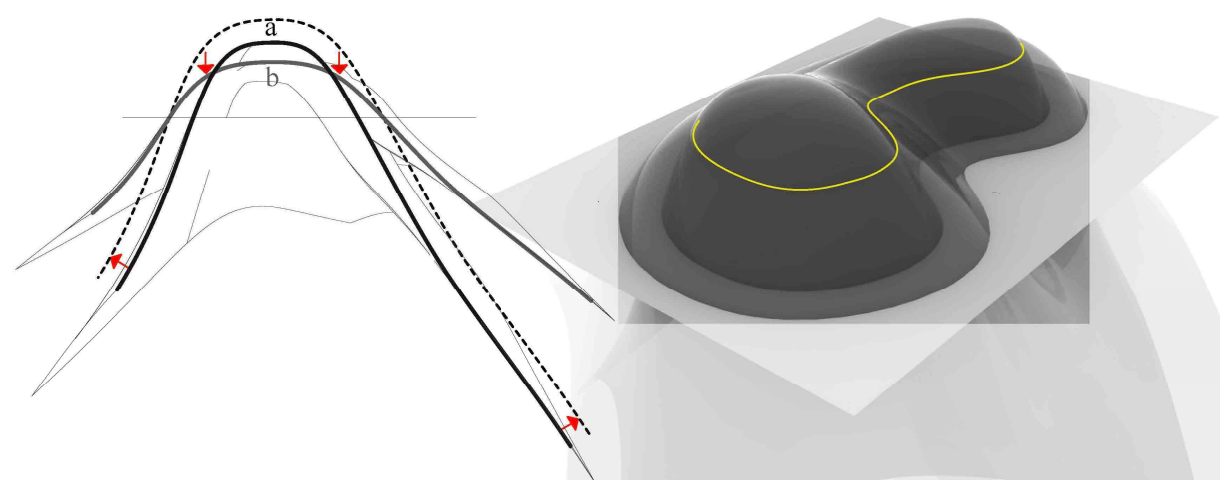

Figure 14: Cut front view (A) and Aerial view (B) of the intersection of the reference surface with a homothetic one

It can be seen in figure 15 that the 3D scaling of the reference surface reduces the global minimum curvature of the surface excepted on the inverted region between the two domes. In this region the minimum radius value increases. The maximum curvature radius increases at the same time on all other regions. It must also be noticed that the intersection line connects the high curvature regions of the both surfaces.

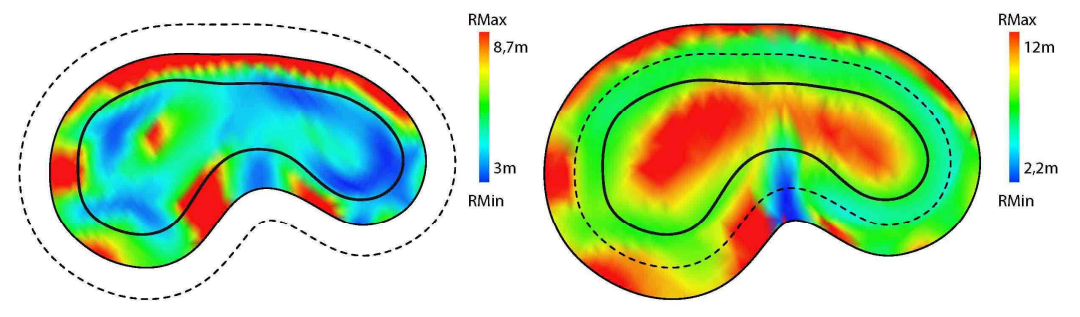

Figure 15: Minimum radius of the reference surface and the intersecting 
Figure 16 shows then different views of the interconnected gridshell with like previously: (A) inside meshing, (B) outside meshing, (C) inside meshing parallel perspective, (D) outside meshing parallel perspective.
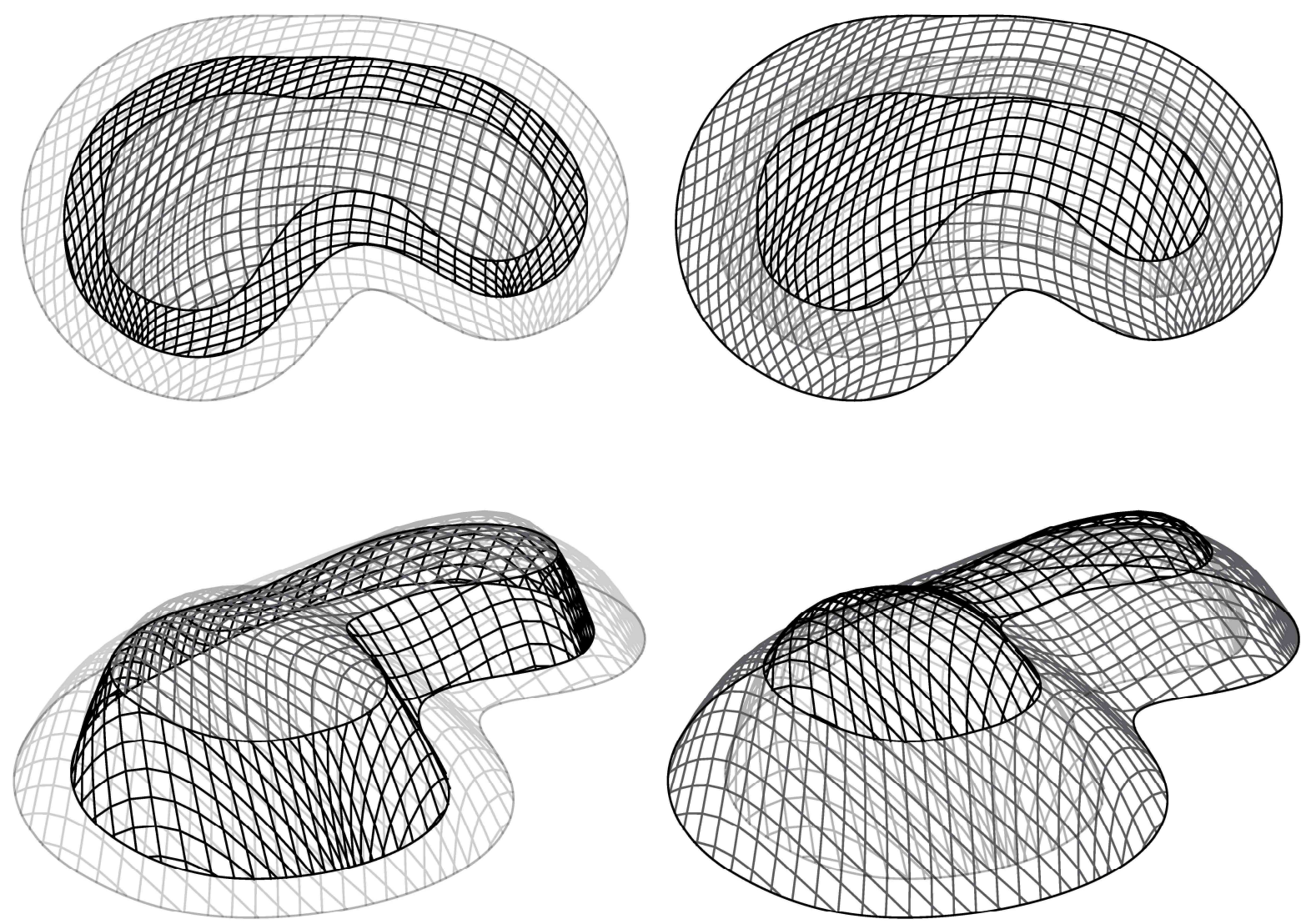

Figure 16: Meshing of the intersecting surfaces

\subsection{Interconnection of the reference gridshell with its mirror image}

In last example, the two patch surfaces are the same than the one presented in the case study 3.1 with the interconnection of two identical gridshells but this time the translation of both surfaces is done in two opposite directions with a mirror effect (figure17). The intersection of both surfaces creates three intersection lines that are approximately located in the regions with large radii of curvature. This results in an architecturally interesting space splitting and also in a reinforcement of the structure in the areas where it is potentially the weakest (from a mechanical point of view). 

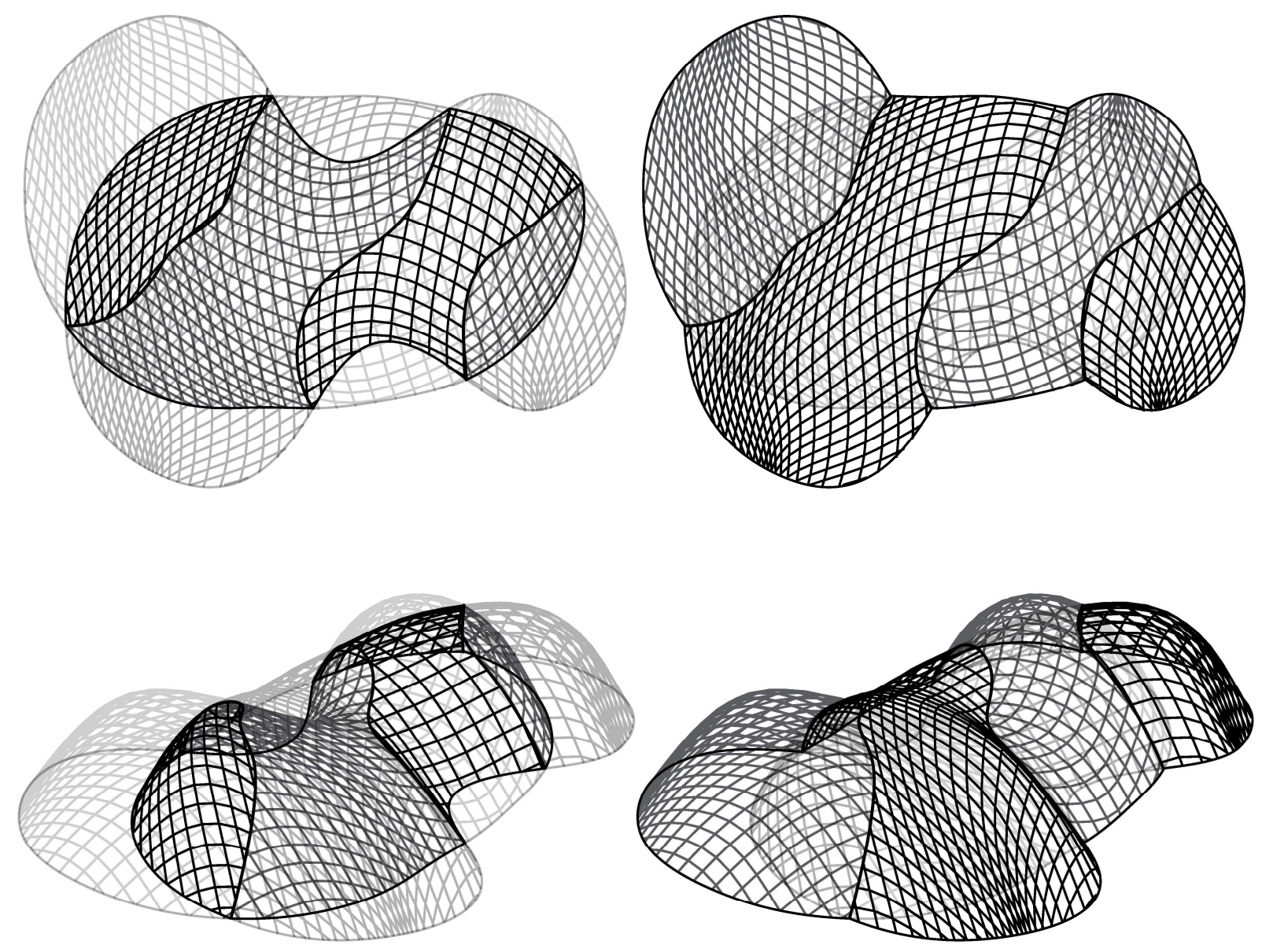

Figure 17: Meshing of the intersecting surfaces

\subsection{Mechanical behavior}

The four variants of interconnected gridshells have then been submitted to the same loading as the reference gridshell in section 2.4 by simply adapting the snow load, so that only external nodes are loaded. For all variants, the interconnection improved the performance of the initial structure, but not in the same way (see table1) and only the last proposal provided a really efficient solution. Indeed in the first three variants works, the additional gridshell provided a kind of support at the ridge of the initial structure and reciprocally. This could not harm the structural behavior of the whole but did not reinforce directly the weakest areas. On the contrary, in the last variant, the mirror gridshell crossed the three weakest area indentified in figure 7 , so that it directly reinforced the structure where needed. When interconnecting gridshells, it is thus essential to identify flat area and to seek to locate interconnections in these areas.

\begin{tabular}{|l|c|c|c|c|c|}
\hline & Reference & Variant 1 & Variant 2 & Variant 3 & Variant 4 \\
\hline Max horiz depl & $7.2 \mathrm{~cm}$ & $3.2 \mathrm{~cm}$ & $5.1 \mathrm{~cm}$ & $2.4 \mathrm{~cm}$ & 0.25 \\
\hline Max vert; depl. & $6.4 \mathrm{~cm}$ & $3.8 \mathrm{~cm}$ & $4.5 \mathrm{~cm}$ & $2.4 \mathrm{~cm}$ & 0.28 \\
\hline $1^{\text {st }}$ critical load & 4.9 & 5.8 & 5.0 & 6.6 & 18.2 \\
\hline
\end{tabular}

Table 1: Comparison of main characteristic of the mechanical behavior of variants. 


\section{Construction of a prototype}

\subsection{Interconnection of two gridshells}

In this section, the feasibility of such structures is illustrated through the construction of a prototype which will be based on the intersection of two simple surfaces like in section 3.3 (see figure 18). The surfaces $a$ and $b$ are a patch of two half ellipsoids. The interior patch surface is intersected with a larger and lower altimetry patch surface. On the ground, the distance for the offset is limited at one meter. The curvature radii of the prototype surfaces are then illustrated in figure 19 (where the black line is the intersection line between the two surfaces). For the inner surface, we have $R_{\min }=1.8 \mathrm{~m}$ and $R_{\max }=3 \mathrm{~m}$, for the outer surface $\mathrm{R}_{\min }=2.7 \mathrm{~m}$ and $\mathrm{R}_{\max }=5.2 \mathrm{~m}$.

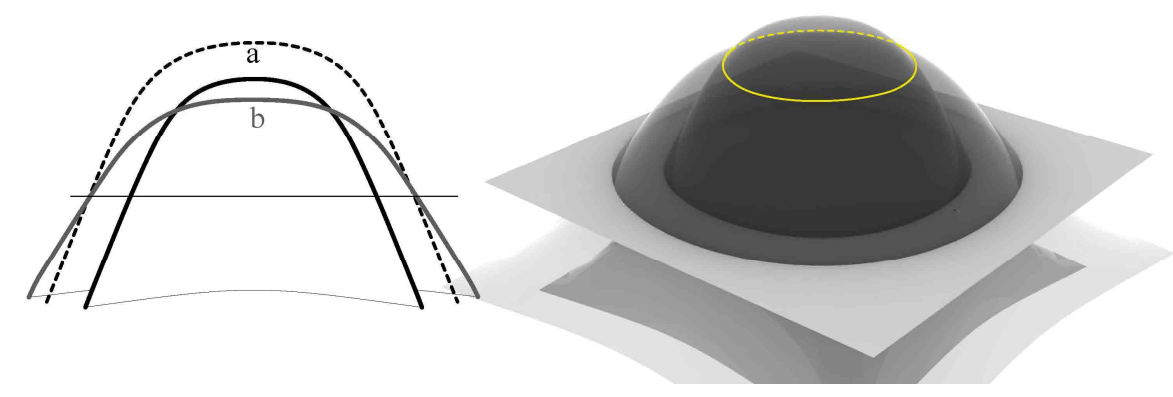

Figure 18: Cut front view (A) and Aerial view (B) of the prototype two surfaces $a$ and $b$
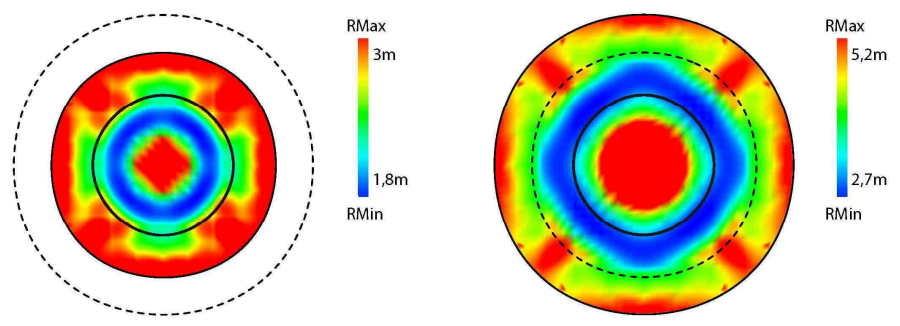

Figure 19: Top view minimum radius of the inner and outer surfaces

Both Grids are meshed with the "compass method" with perpendicular director polylines crossing and lying on top of the patch surfaces. To facilitate the interweaving of the two grids, the director polylines for the meshing of the first patch surface are fixed with an angle of $45^{\circ}$ to the grid director polylines for the meshing of the second patch surface (see figure 20).
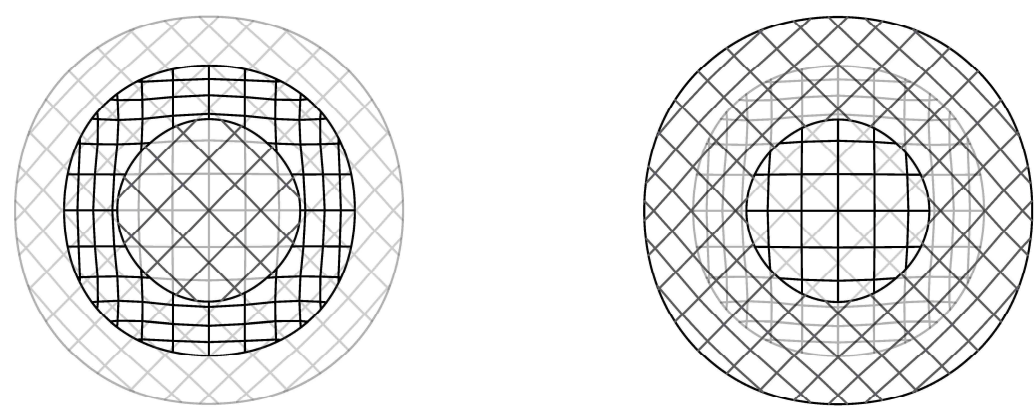

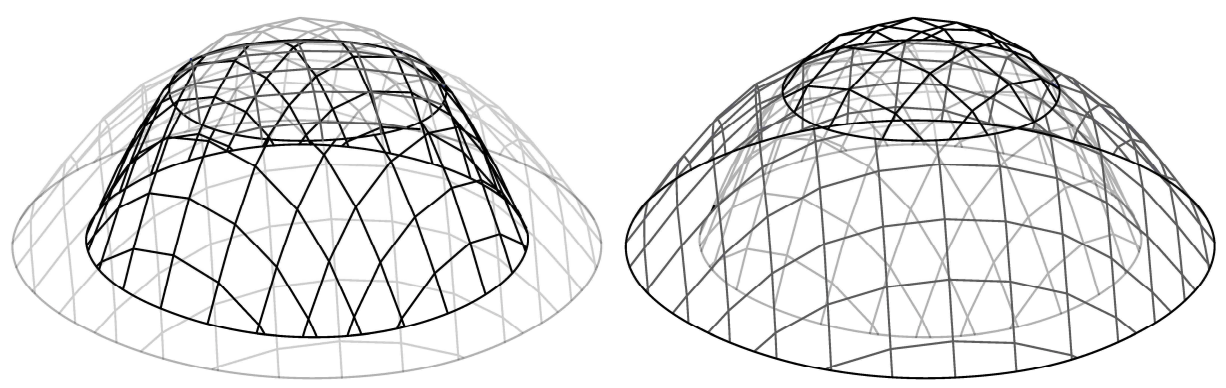

Figure 20: Meshing of the intersecting surfaces

\subsection{Construction of the double Grid}

The challenge was to build the two Grids on the ground. To facilitate the erection of the two interweaving grids (see figure 21) the mesh width is wider than in the model shown in figure 20 . Indeed, initially the mesh width was set at 0,8 meters but finally ended up with a mesh width of 1,6 meters.
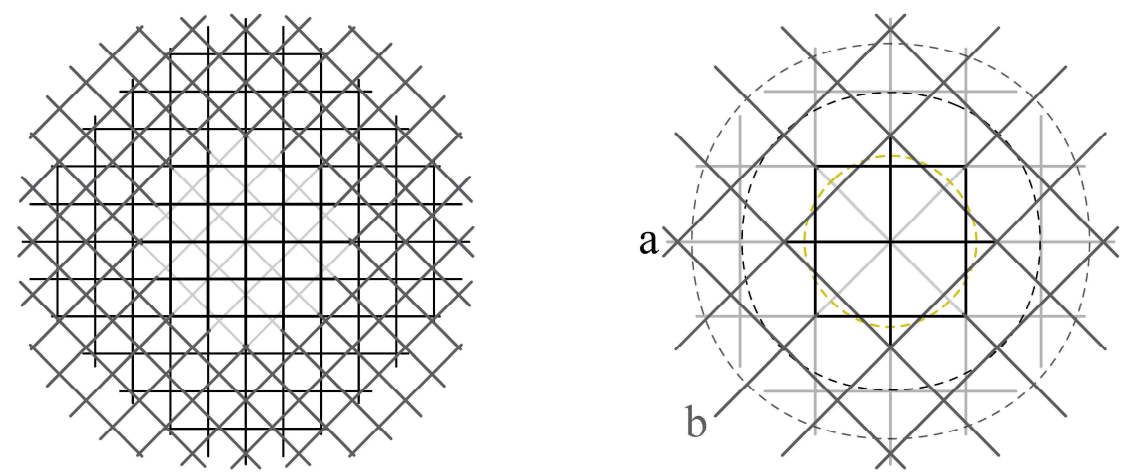

Figure 21: Meshing plan of the double Grid on ground before

Practically, both grids were assembled on the ground, using for two different colors for the two surfaces: white for the larger one, blue for the highest one (see figure 22). All connections between members were made with standard scaffolding elements, including connections between the blue beams of the interior gridshell with the exterior white beams of the larger gridshell. Both grids were assembled interwoven, then lifted and finally connected between them. Following, an experimental test of the connection between both gridshells with a local load on the top of $60 \mathrm{~kg}$ was conducted and showed that the structure is more rigidwith than without connection. The last step of the construction - that has not been done for this prototype but that is mandatory for a real gridshell structure- should be to stabilize the two meshed forms with a third direction of beams or with stiffening cables in both directions to block the network of parallelograms.

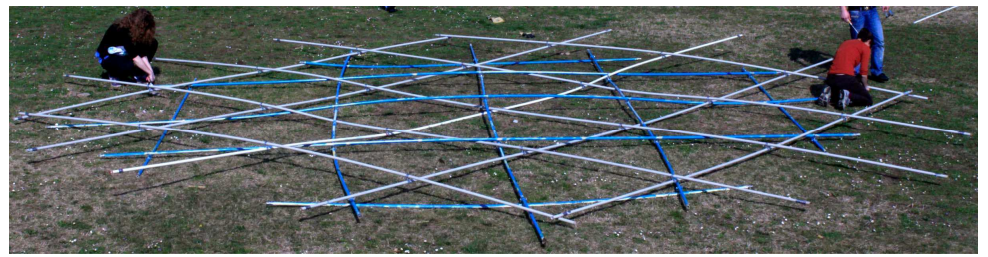




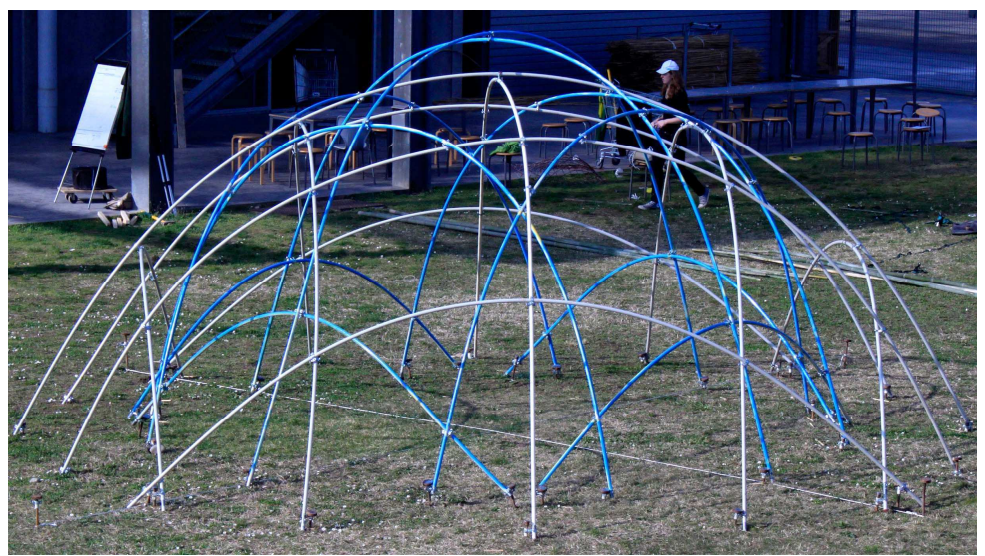

Figure 22: View of the interweaving grid on the ground and of the two interconnected gridshells

\subsection{Roofing of the structure}

A roofing was finally realized atop of the exterior beams with an elastic membrane. The distance between the membrane and the inner space - limited by the interior beams emphasize astonishingly the lightness of the structure. The possibility of placing the roofing wherever wanted on the two grids offers a new field in the conception of gridshells.
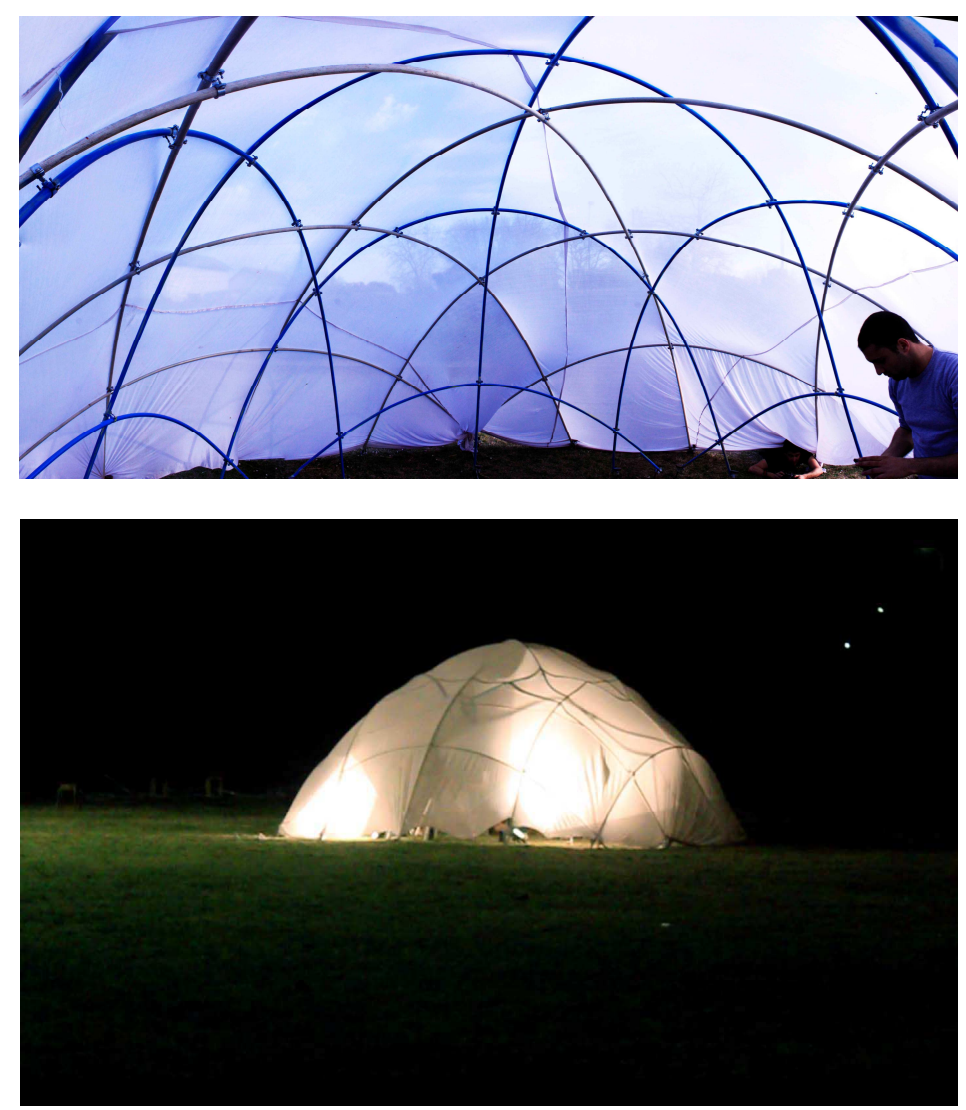

Figure 23: Interior view and exterior view by night of the prototype

\section{Conclusion}

In this paper, an investigation of interconnection of gridshells was presented. After showing the method to control the form and the mesh, different configurations of 
interconnections of gridshells were proposed. The structural behaviour of the various configurations was compared with the initial structure without interconnections. It showed that if the intersection lines cross the area with the lower curvature of the initial structure, then interconnection provides an efficient alternative way to improve the performance of a gridshell. A report on the experimental prototype of interconnected gridshell was also presented. The construction showed that the erection process is more complex than a normal gridshell. It seems thus that interconnection of gridshell has a good potential in the development of a new generation of gridshells with astonishing interior spaces.

\section{Acknowledgments}

We would like to thank Jean-François Caron and Frederic Tayeb from the Navier laboratory. For the construction of the prototype we would like to thank Gilles Ebersolt and his master class students Johanna Schmidt, Florentia Christokorov, Mehdi Grosjean, Amine Khodri, Tra Lehuong, Florentin Duroy, Maureen Gerbe, Emilie Anglada, Habib Bencherif, Amir Hossein Shirvani, Maxime Ravaux, Martin Delarue, Quentin Leteurtre and Pauline Mourani (ENSAV). For the prototype construction we also would like to thank the technical team of "Les Grands Ateliers" and the students of ENPC Juliette Adam and Camille Glacet.

\section{References}

[1] Baverel O, Caron J-F, Tayeb F, Peloux L (2012), Gridshells in composite materials: Construction of a $300 \mathrm{~m} 2$ forum for the solidays' festival in Paris, Structural Engineering International: Journal of IABSE, v 22, n 3, p 408-414

[2] Douthe C., Baverel O., and Caron J-F., (2010) Gridshell structures in glass fibre reinforced polymers, Construction and Building Materials, v 24, n 9, p 1580-1589

[3] Douthe C., Baverel O., and Caron J-F., (2007) Gridshell in composite materials: toward wide span shelters, Journal of the International Association for Shell and Spatial structures, 48(155): p.175-180

[4] Douthe C., Baverel O., and Caron J-F., (2006) Form-finding of a grid shell in composite materials, Journal of the International Association for Shell and Spatial structures, 40: p. 53-62

[5] Douthe C., Study of slender prestressed structures in composite materials: application to the conception of gridshells, PhD thesis, ENPC (French), 2007:p.274

[6] Rhinoceros, Nurbs modelling for Windows, http://www.rhino3d.com/5/help/commands.html

[7] Frei O. et al, IL10 Gitterschalen, Institut für leichte Flächentragwerke (IL), 1974

[8] Knight T.W. (1994), Shape grammars and color grammars in design, Environment and Planning B: Planning and Design, 21(6), pp. 705-735 